\title{
Eosinophilic Mastitis: A Case Report
}

\author{
Hyewon Bang, M.D. ${ }^{1}$, Kwan Ho Lee, M.D., Ph.D. ${ }^{1}$, Hyeon Sook Kim, M.D., Ph.D. ${ }^{2}$, Ki Ouk Min, M.D., Ph.D. ${ }^{3}$, \\ Seung Hye Choi, M.D., Ph.D. ${ }^{1}$ \\ Departments of ${ }^{1}$ Surgery, ${ }^{2}$ Radiology, ${ }^{3}$ Pathology, The Catholic University of Korea, Eunpyeong St. Mary Hospital, Seoul, Korea
}

\begin{abstract}
Eosinophilic mastitis is a rare disease in which eosinophils infiltrate the ducts and lobules of the breast, causing mammary inflammation. Although eosinophilic mastitis is a benign condition, it presents similarly to and is often mistaken for breast cancer. Here we report the first case of eosinophilic mastitis in Korea. A 43-year-old woman presented with redness and swelling in her right breast. A breast ultrasound revealed ill-defined isoechoic and hyperechoic areas in the upper half and lower inner quadrant of the right breast combined with axillary level I lymphadenopathy. A histopathological examination by core needle biopsy demonstrated lymphoplasma cell infiltration with eosinophils. A peripheral blood examination revealed an eosinophil count of $5,000 / \mathrm{mm}^{3}$. The patient was treated with oral steroids, antibiotics, and leukotriene receptor antagonists. Four months later, the eosinophil count had returned to normal, and breast ultrasound exhibited marked improvement.
\end{abstract}

Key Words: Breast, Eosinophilia, Mastitis

\section{INTRODUCTION}

Eosinophilic mastitis is a rare disease in which eosinophils infiltrate the ducts and lobules of the breast, causing mammary inflammation [1]. Although the clinical features, imaging findings, and physiological features are nonspecific, they are similar to those of breast cancer; therefore, it is often mistaken for breast cancer [2].

Eosinophilic mastitis is not a common disease and has not been reported in Korea to date. Here we report the diagnosis and treatment of a case of eosinophilic mastitis. To the best of our knowledge, this is the first reported case of eosinophilic mastitis in Korea.

\section{CASE REPORT}

In June 2019, a 43-year-old woman presented with redness and swelling of her right breast that had started approximately 2 months prior. She was diagnosed with bronchial asthma at the age of 23 years and was recently treated in a hospital for asthma-related symptoms. The patient was allergic to penicillin and doxycycline. Her family history was unclear. She had a history of smoking a pack of cigarettes per

Correspondence: Seung Hye Choi, M.D., Ph.D.

Department of Surgery, The Catholic University of Korea, Eunpyeong St. Mary Hospital, 1021 Tongil-ro, Eunpyeong-gu, Seoul 03312, Korea Tel: +82-2-2030-4646, Fax: +82-2-2030-4647, E-mail: gsshchoi@catholic.ac.kr Received: Jan 4, 2021 Revised: Jan 29, 2021 Accepted: May 20, 2021 day from 23 to 35 years of age, at which time she quit smoking. She had menarche at the age of 15 years and was gravida 1, para 0 . A loop electrosurgical excision procedure was performed for cervical intraepithelial neoplasia grade 2 with myoma in 2008, and a levonorgestrel-releasing intrauterine device was inserted in 2013 because of the myoma. A physical examination revealed swelling and redness of the right breast that was firm to the touch. The results of mammography performed in April 2019 at another hospital were also evaluated. Mammography images show global asymmetry of the central portion of the right breast craniocaudal and mediolateral oblique views relative to the corresponding area of the left breast (Figure 1)

In our hospital, breast ultrasonography was performed for diagnosis. Breast ultrasound revealed ill-defined isoechoic and hyperechoic areas in the upper half and lower inner quadrant of the right breast along with an edematous change in the subcutaneous fat and dilated lymphatics combined with axillary level I lymphadenopathy (LAP) (Breast Imaging-Reporting and Data System; BI-RADS 4C) (Figure 2A, B). Color Doppler breast ultrasound showed an increase in vascular pattern (Figure 2C). To exclude malignant disease and ensure an accurate diagnosis, core needle biopsy of the right breast and right axilla was performed using an 18-gauge needle under local anesthesia. Histopathological examination revealed infiltration of lymphoplasma cells with eosinophils (Figure 3). Reactive hyperplasia with eosinophils was observed in the right axilla. Chest computed tomography showed 

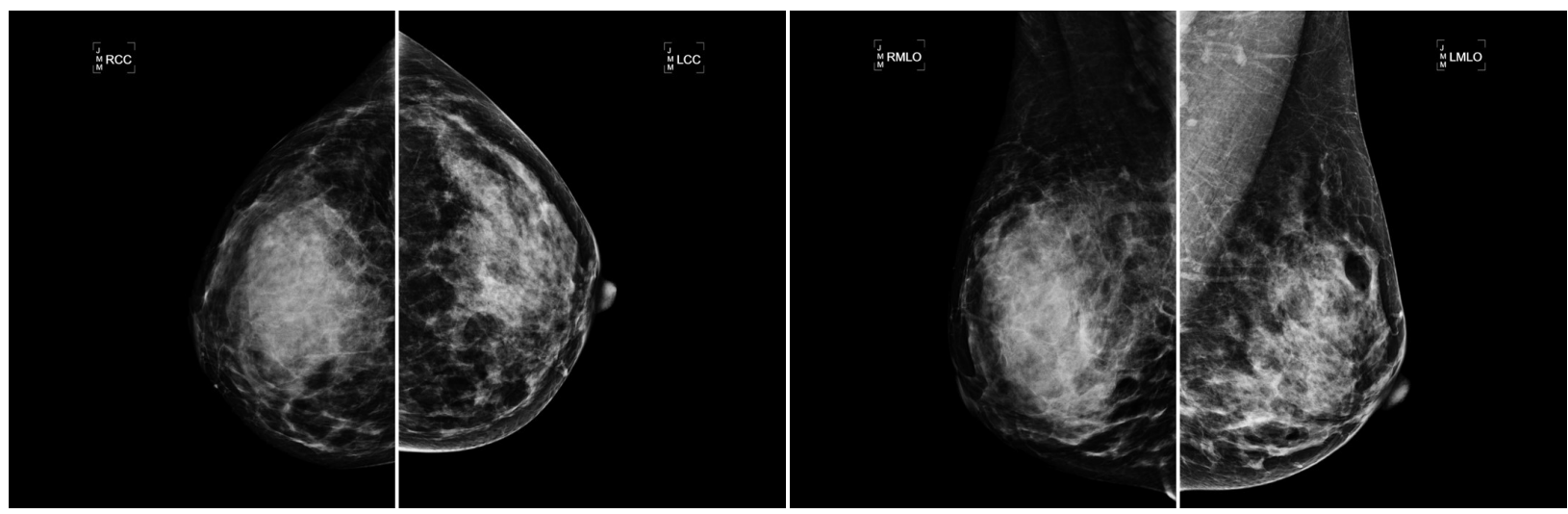

Figure 1. Mammography images show the global asymmetry occupying the central portion of the right breast craniocaudal and mediolateral oblique view relative to the corresponding area of the left breast.
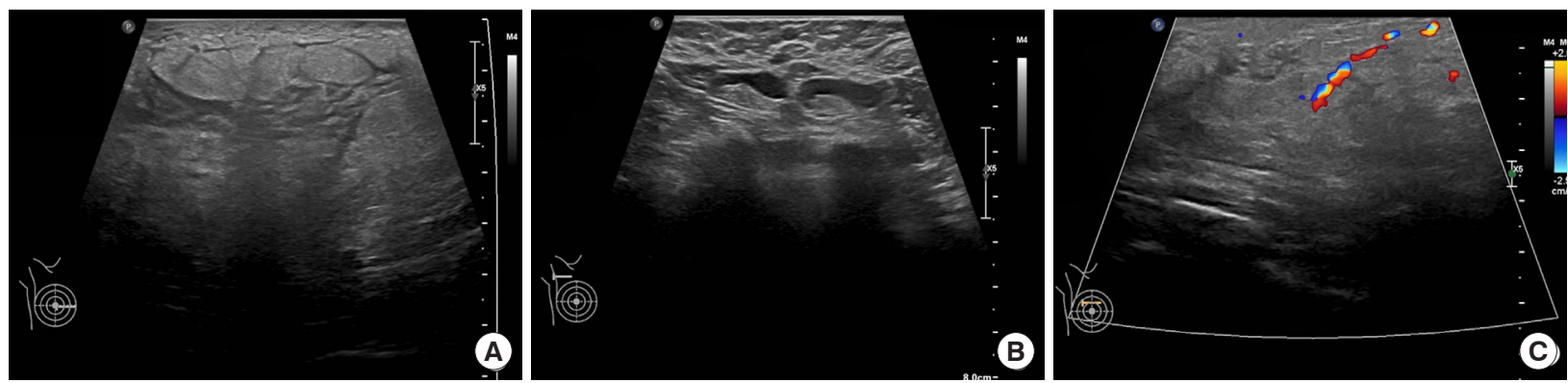

Figure 2. Breast ultrasound showing ill-defined isoechoic and hyperechoic areas in the upper half and lower inner quadrant of the right breast (A) combined with axillary level I lymphadenopathy (B). An increase in the vascular pattern is noted (C).

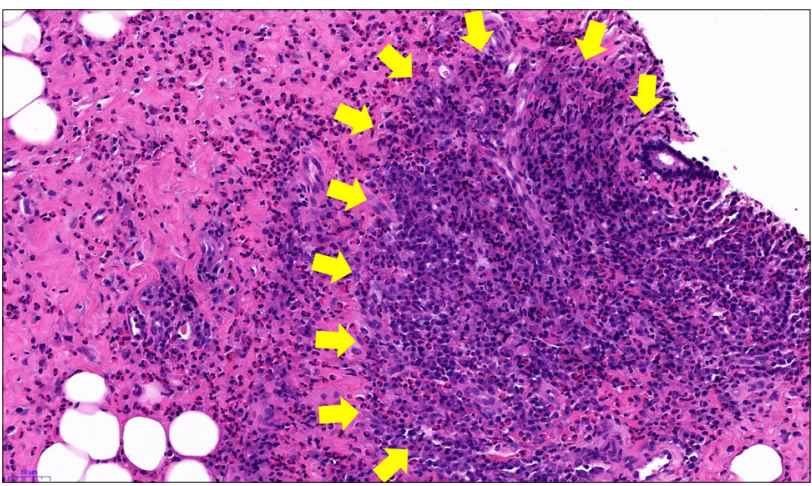

Figure 3. A histopathological examination showed an infiltration of lymphoplasma cells with eosinophils (hematoxylin and eosin stain, $\times 20$ ).

marked swelling of the right breast (Figure 4). A peripheral blood examination revealed a total leucocyte count of $12,000 / \mathrm{mm}^{3}$ (normal range, $4,000-10,000 / \mathrm{mm}^{3}$ ) with $33 \%$ eosinophils (normal range, 1$5 \%$ ), and an eosinophil count of $5,000 / \mathrm{mm}^{3}$ were observed. Hepatitis $B$ surface antigen and anti-hepatitis $C$ virus antibodies were negative. Further investigations were performed after a histopathologic diag-

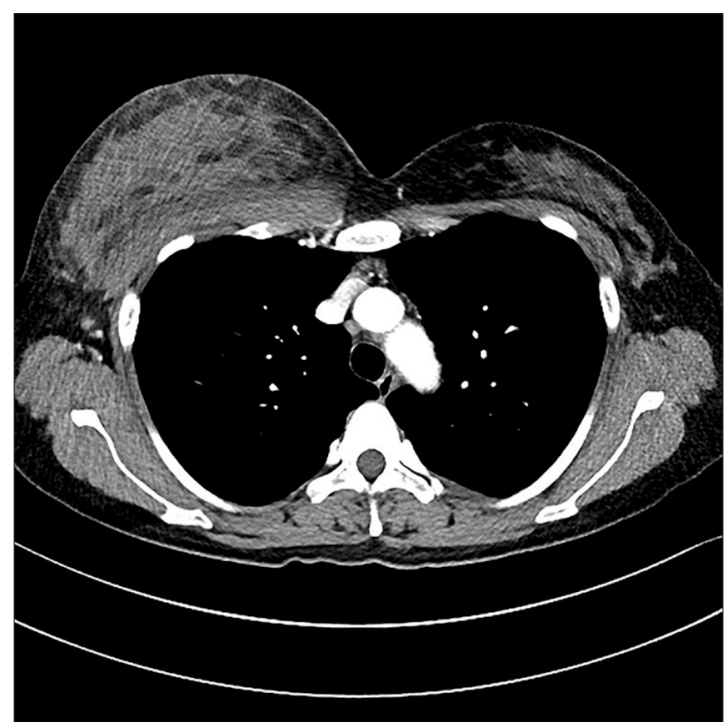

Figure 4. Chest computed tomography image showed prominent swelling of the right breast.

nosis of eosinophilic mastitis was made. Breast magnetic resonance imaging revealed extreme fibroglandular tissue and mild non-mass 


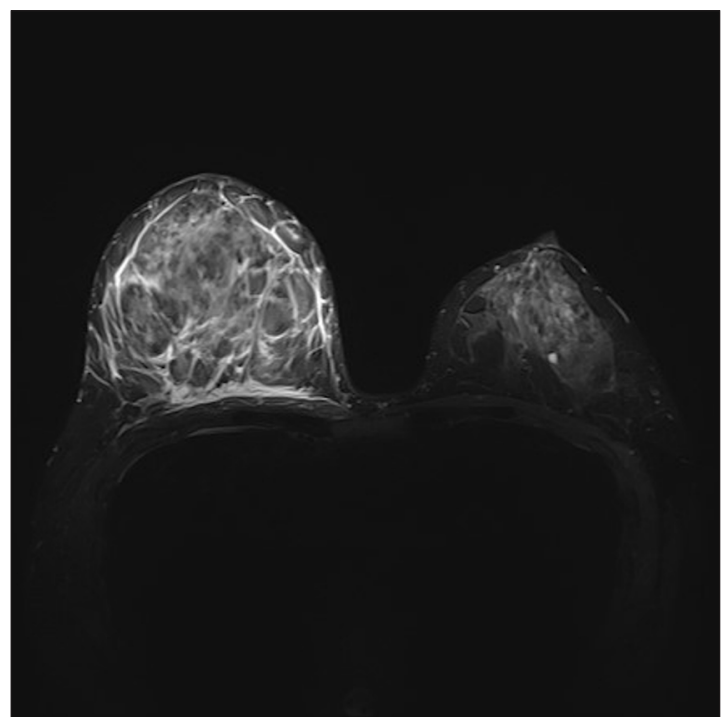

Figure 5. Magnetic resonance image revealed extreme fibroglandular tissue and mild non-mass enhancement along with increased vascularity and diffuse edematous changes in the right breast parenchyma.

enhancement along with increased vascularity and diffuse edematous changes in the right breast parenchyma (Figure 5). These findings were accompanied by a right axillary level I LAP. There were no specific findings on chest radiography. However, a chest computed tomography scan showed a wedge-shaped consolidation of the right middle lobe, lingular division, and left lower lobe of the lung. Bilateral maxillary sinusitis was observed on the Waters and Caldwell radiographic views. Pulmonary function test findings were consistent with asthma with an obstructive pattern. The immunoglobulin E level was $162.7 \mathrm{IU} / \mathrm{mL}$ (normal value, below $87 \mathrm{IU} / \mathrm{mL}$ ), fibrinogen was $423 \mathrm{mg} /$ $\mathrm{dL}$ (normal range, $173-372 \mathrm{mg} / \mathrm{dL}$ ), C-reactive protein was $0.78 \mathrm{mg} /$ $\mathrm{dL}$ (normal range, $0.01-0.5 \mathrm{mg} / \mathrm{dL}$ ), and antineutrophil cytoplasmic antibodies were negative.

Treatment was initiated after the diagnosis of eosinophilic mastitis with asthma was confirmed. Starting on June 21, 2019, the patient received oral steroids at a dose of $60 \mathrm{mg} / \mathrm{day}$ along with antibiotics and a leukotriene receptor antagonist for approximately 14 days. Additionally, the oral steroids were tapered from $60 \mathrm{mg} /$ day to $5 \mathrm{mg}$ /day over the next 5 months. After the start of the treatment, the size of the breast decreased and the redness improved; however, intermittent mastalgia persisted. In the initial 2 weeks, complete blood count and eosinophil count were performed once a week, revealing a white blood cell count of $8,000-12,700 / \mathrm{mm}^{3}$ (normal range, $4,000-10,000 / \mathrm{mm}^{3}$ ), eosinophils of $0.1 \%-1.5 \%$ (normal range, $1 \%-5 \%$ ), and eosinophil count of $0-200 / \mathrm{mm}^{3}$. After two weeks, follow-up was performed at monthly intervals. Subsequently, $0.5 \%-3.1 \%$ eosinophils (normal range, $1 \%-5 \%$ ) and eosinophil count of $100-300 / \mathrm{mm}^{3}$ were observed. Four months later, the eosinophil count was returned to normal levels.

In October 2020, mammography and breast ultrasound showed marked improvement compared to the previous ultrasound in June 2019. No definite abnormality is noted in the breast and axillary regions on mammography and the ill-defined isoechoic and hyperechoic areas had disappeared and a tiny cyst with a $0.7-\mathrm{cm}$ circumscribed ovoid isoechoic nodule in the subareolar area was observed. This case study was approved by our Institutional Review Board (IRB No. PC21ZASI0025).

\section{DISCUSSION}

Eosinophilic mastitis is a very rare condition worldwide for which only nine cases have been reported to date. Eosinophilic mastitis is a phenomenon in which eosinophils cause mammary gland inflammation and are usually accompanied by high eosinophilia. To date, only one case of eosinophilic mastitis without peripheral eosinophilia has been reported [1], and all others demonstrated peripheral eosinophilia. Eosinophilia is observed in asthma, Churg-Strauss syndrome, hypereosinophilic syndrome (HES), parasitic infections, collagen vascular diseases, and hematological malignancies. The majority of cases reported worldwide have been associated with diseases such as asthma and Churg-Strauss syndrome. At the 2011 Eosinophil Disorders and Syndromes working conference, experts from various fields gathered to organize the concepts and terminology related to hypereosinophilia and develop a new taxonomy [3]. First, hypereosinophilia (HE) is diagnosed by peripheral blood tests when more than 1,500 eosinophils/ $\mu \mathrm{L}$ are detected at least twice every 4 weeks, or when biopsy results show tissue HE. Second, hypereosinophilic syndrome (1) satisfies the definition of HE, (2) involves tissue damage and/or dysfunction due to tissue HE, and (3) is defined as the absence of other major causes of organ damage. However, since the tissue-damaging effects of eosinophilia are often unclear in a single organ, such cases are not classified as HES but as eosinophil-associated single-organ diseases. These include eosinophilic mastitis, pulmonary eosinophilic syndrome, and eosinophilic gastroenteritis [3]. 
The reported age range of eosinophilic mastitis cases is 30 s to $60 \mathrm{~s}$ years. Symptoms commonly included nipple discharge and a breast lump with or without pain $[1,2,4-8]$. Imaging findings were nonspecific, and mammography mainly showed asymmetric nodular shadows, dense parenchymal patterns, and ill-defined masses [2,4]. On breast ultrasound, a large solid or heterogeneous mass was predominantly observed, and the echogenicity of the breast parenchyma was increased. Skin thickening and duct dilatation were also observed. Although each reported case showed slightly different symptoms, imaging, and peripheral eosinophilia, aspiration or core needle biopsy is commonly used to diagnose eosinophilic mastitis. Treatment typically includes steroids, and the goal is to reduce the number of eosinophils in the blood and tissues, thereby reducing the direct and indirect damage caused by eosinophil-derived substances. In addition, a very rare case of eosinophilic mastitis was reported that, improved with anti-allergic drug administration [4]. Based on this case, if the mastitis is confined to the breast without eosinophilic damage to other organs, it is possible to treat it with anti-allergic drugs. Although eosinophilic mastitis is a very rare disease, it is essential to differentiate it from breast cancer to ensure the accurate diagnosis and treatment of such patients. In our case, breast lumps appeared clinically and radiologically in asthmatic patients with peripheral eosinophilia. In such cases, even if the histological malignancy is not diagnosed, it may be considered malignant and subject to unnecessary surgery. Thus, the high suspicion in the physician's mind and use of a histological examination and laboratory tests may enable an accurate diagnosis and treatment of malignant and benign breast disease. In addition, it is important not to overlook various types of mastitis through a careful history taking. This allows us to provide more accurate and high-quality medical care to patients.

\section{CONFLICT OF INTEREST}

The authors declare that they have no competing interests.

\section{REFERENCES}

1. Parakh A, Arora J, Srivastava S, Goel RK. Isolated eosinophilic infiltration of the breast. Indian J Radiol Imaging 2016;26:383-5.

2. Singh A, Kaur P, Sood N, Puri H, Garg B. Bilateral eosinophilic mastitis: an uncommon unheard entity. Breast Dis 2015;35:33-6.

3. Valent P, Klion AD, Horny HP, Roufosse F, Gotlib J, Weller PF, et al. Contemporary consensus proposal on criteria and classification of eosinophilic disorders and related syndromes. J Allergy Clin Immunol 2012;130:607-12.

4. Takahashi K. A very rare case of eosinophilic mastitis. Int J Surg Case Rep 2018;49:251-4.

5. Garg M, Kumar S, Neogi S. Eosinophilic mastitis masquerading as breast carcinoma. J Surg Case Rep 2012;2012:1.

6. Komenaka IK, Schnabel FR, Cohen JA, Saqi A, Mercado C, Horowitz E, et al. Recurrent eosinophilic mastitis. Am Surg 2003;69: 620-3.

7. Villalba-Nuno V, Sabate JM, Gomez A, Vidaller A, Catala I, Escobedo A, et al. Churg-Strauss syndrome involving the breast: a rare cause of eosinophilic mastitis. Eur Radiol 2002;12:646-9.

8. Thompson AB, Barron MM, Lapp NL. The hypereosinophilic syndrome presenting with eosinophilic mastitis. Arch Intern Med 1985;145:564-5. 